\title{
ON EFFECTIVE MODULI OF AN ELASTIC BODY CONTAINING PERIODICALLY DISTRIBUTED VOIDS: COMMENTS AND CORRECTIONS*
}

\author{
BY \\ S. NEMAT - NASSER ( Northwestern University) \\ AND \\ M. TAYA (University of Delaware)
}

In [1] the present writers proposed a general method for estimating the stress and strain fields in an elastic body containing periodically distributed voids. The basic formulation, as well as the solution methods, given in Secs. 1 to 3 , is for the case when the overall strains are prescribed to be $\varepsilon^{0}$. In this case the overall (average) stresses are defined by

$$
\bar{\sigma}_{i j}=C_{i j k l}^{*} \varepsilon_{k l}^{0},
$$

where $\mathbf{C}^{*}$ is the overall elastic modulus tensor.

If, on the other hand, the overall (average) stresses are prescribed to be $\sigma^{0}$, then the overall (average) strains, $\bar{\varepsilon}$, become

$$
\bar{\varepsilon}_{i j}=D_{i j k l}^{*} \sigma_{k l}^{0},
$$

where $\mathbf{D}^{*}$ is the overall compliance tensor which, for consistency, must (and does) equal the inverse of $\mathbf{C}^{*}$; see Nemat-Nasser at al. [2].

In our early work [1], we calculated the average strain energy (per unit volume) to be

$$
(1 / 2) D_{i j k l} \sigma_{i j}^{0} \sigma_{k l}^{0}+(1 / 2) f \sigma_{i j}^{0} \bar{\varepsilon}_{i j}^{*},
$$

when the overall stresses are prescribed. This then must equal $(1 / 2) \sigma_{i j}^{0} \bar{\varepsilon}_{i j}=$ $(1 / 2) D_{i j \mathbf{k} l}^{*} \sigma_{i j}^{0} \sigma_{k l}^{0}$, from (2), so that $\mathbf{D}^{*}$ is defined by

$$
D_{i j k l}^{*} \sigma_{i j}^{0} \sigma_{k l}^{0}=D_{i j k l} \sigma_{i j}^{0} \sigma_{k l}^{0}+f \sigma_{i j}^{0} \bar{\varepsilon}_{i j}^{*}
$$

which is Eq. (4.4) of [1], where $f$ is the volume fraction of voids. The average transformation strain, $\bar{\varepsilon}^{*}$, in (4) must, however, be obtained for the case when the stresses (and not the strains) are regarded to be prescribed. Therefore, in the integral equation (2.13) of [1], $\varepsilon_{k l}^{0}$ must be replaced by $\bar{\varepsilon}_{k l}$ defined by (2) above, if Eq. (4) is used to calculate $\mathbf{D}^{*}$. For this reason the overall moduli given in Tables 1 to 6 , as well as in Figs. 1 and 2 of [1], are

\footnotetext{
* Received January 16, 1984.
} 
incorrect. Note that the corresponding values of the average transformation strains, $\bar{\varepsilon}^{*}$, in Tables 1, 2, 4, and 5 of [1] are correct, and may be used to estimate the overall elastic moduli from the following equation [2]:

$$
C_{i j k l}^{*} \varepsilon_{i j}^{0} \varepsilon_{k l}^{0}=C_{i j k l} \varepsilon_{i j}^{0} \varepsilon_{k l}^{0}-f \sigma_{i j}^{0} \bar{\varepsilon}_{i j}^{*}
$$

which corresponds to the case when the overall strains are prescribed to be $\varepsilon^{0}$; in (5), $\sigma_{i j}^{0}=C_{i j k l} \varepsilon_{k l}^{0}$ (for details see [2]).

Equations (4.7), (4.8), and (4.9) of [1] should be changed to:

$$
\begin{aligned}
K^{*} / K & =1-f \bar{\varepsilon}^{*} / \varepsilon^{0}, \\
\mu^{*} / \mu & =1-f \bar{\varepsilon}_{12}^{*} / \varepsilon_{12}^{0}, \\
\mu^{*} / \mu & =1-f \bar{\varepsilon}^{*} / \varepsilon^{0},
\end{aligned}
$$

where the values of the transformation strain components are given in Tables 1, 2, 4, and 5 of [1]. To obtain the corrected overall moduli from the incorrect values given in tables in [1], use the following expression:

$$
\left[X^{*} / X\right]_{\text {corrected }}=2-1 /\left[X^{*} / X\right]_{T}, \quad X=K, \mu,
$$

where the right-hand quantity in (6), denoted by the subscript $T$, is the value of the corresponding modulus given in Tables 1 to 6 and Figs. 1 and 2 of [1].

In [2], Nemat-Nasser et al. present detailed analytical and numerical results for porous elastic solids, including the induced anisotropy due to the periodic nature of the void distribution, and compare results with experimental data. In [3], Iwakuma and NematNasser give results for elastic solids containing periodically distributed ellipsoidal elastic inclusions and voids.

\section{BIBLIOGRAPHY}

[1] S. Nemat-Nasser and M. Taya, On effective moduli of an elastic body containing periodically distributed voids, Quarterly Applied Math. 39 43(1981)

[2] S. Nemat-Nasser, T. Iwakuma, and M. Hejazi, On composites with periodic structure, Mechanics of Materials 1, 239(1982)

[3] T. Iwakuma and S. Nemat-Nasser, Composites with periodic microstructure, Computers \& Structures 16, 13(1983) 